\title{
A SURVEY OF MEDICINAL PLANTS OF WADI AL-KOUF IN AL-JABAL AL-AKHDAR, LIBYA
}

\author{
Manal Al-Traboulsi* \& Mohamed A. Alaib \\ Botany Department, Faculty of Sciences, University of Benghazi, Benghazi, Libya
}

\begin{abstract}
Al-Traboulsi, M. \& Alaib, M. A.: A survey of medicinal plants of Wadi Al-Kouf in Al-Jabal Al-Akhdar, Libya. Nat. Croat., Vol. 30, No. 2, 389-404, 2021, Zagreb.

Al-Jabal Al-Akhdar (the Green Mountain) of Cyrenaica, Libya is known to have the highest plant species diversity in the country, particularly in the depressions and the valleys of the mountain. Wadi Al-Kouf is the largest valley in the mountain, with a rich flora of aromatic and medicinal plants. The present investigation was conducted to record and list the medicinal plant species growing in the valley during the flowering season of 2019. Results revealed that Wadi Al-Kouf hosts 107 medicinal plant species belonging to 49 families and 93 genera. Dicotyledons were the most represented group of angiospermae with 38 families, 79 genera and 89 species, whereas Monocotyledons were represented by only 8 families, 10 genera and 13 species. Asteraceae, Lamiaceae, Fabaceae and Solanaceae were the families with the highest number of species; $11,9,6$ and 6 species, respectively. The most dominant life forms found in the valley were Therophytes (25.2\%), Phanerophytes $(25.2 \%)$ and Chamaephytes $(24.3 \%)$, followed by Hemicryptophytes $(15.9 \%)$ and Geophytes $(9.3 \%)$. Nine endemic species were detected and recorded.
\end{abstract}

Keywords: Cyrenaica, endemic flora, ethnobotany, Libyan vegetation, medicinal plants, Mediterranean flora

Al-Traboulsi, M. \& Alaib, M. A.: Istraživanje ljekovitog bilja doline Wadi Al-Kouf planine Al-Jabal Al-Akhdar u Libiji. Nat. Croat., Vol. 30, No. 2, 389-404, 2021, Zagreb.

Al-Jabal Al-Akhdar (Zelena planina) u pokrajini Cirena u Libiji poznata je po najvećoj biljnoj raznolikosti u zemlji, osobito u udolinama i nizinama te planine. Wadi Al-Kouf je najveća udolina u planini, s bogatom florom koja se sastoji od aromatičnog i ljekovitog bilja. Cilj našeg istraživanja tijekom vegetacijske sezone 2019. bilo je zabilježiti i popisati ljekovito bilje koje raste u toj dolini. Rezultati su pokazali da u dolini Wadi Al-Kouf raste 107 vrsta ljekovitog bilja, iz 49 porodica, odnosno 93 roda. Najzastupljenije kritosjemenjače su dvosupnice, s 38 porodica, 79 rodova i 89 vrsta, dok su jednosupnice zastupljene sa samo 8 porodica, 10 rodova i 13 vrsta. Porodice s najviše zabilježenih vrsta bile su Asteraceae, Lamiaceae, Fabaceae i Solanaceae; redom 11, 9, 6 i 6 vrsta. Najdominantniji životni oblici u dolini bili su terofiti $(25,2 \%)$, fanerofiti $(25,2 \%)$ i hamefiti $(24,3 \%)$, zatim hemikriptofiti $(15,9 \%)$ i geofiti $(9,3 \%)$. Zabilježeno je devet endemičnih vrsta.

Ključne riječi: Cirena, endemska flora, etnobotanika, vegetacija Libije, ljekovite biljke, mediteranska flora

\section{INTRODUCTION}

Medicinal plants are the foundation of herbal medicine, aromatherapy, homoeopathy and ayurvedic medicine. Herbal medicine is defined as the use of plant material or plant extract to cure illness, relieve pain or boostoverall health (WHITE \& FosTeR, 2000). To manufacture some conventional pharmaceuticals, certain chemical constitu-

\footnotetext{
*Corresponding author; manal_altraboulsi@yahoo.com, manal.eltraboulsi@uob.edu.ly; phone no. 00218-91-836-0343
} 
ents have to be extracted from medicinal plants. It is estimated that $35 \%$ of all prescription drugs are derived from plants. Cultures and civilizations all over the world have used herbal medicine for thousands of years. The earliest records of herbal medicine were found in China dating back to 2000 B.C. containing around 365 herbal formulas (Lewis \& ELVIN-Lewis, 2003). The use of medicinal plants started to decline in the late $19^{\text {th }}$ century when scientists managed to manufacture synthetic versions of the active ingredients of the medicinal herbs. However, with the growing dissatisfaction with the harmful side effects of modern synthetic medicines, attention to medicinal plants has been renewed and the benefits of using natural plant remedies have been rediscovered (EL-GADI, 1989).

Libyan culture, like every other culture in the world, relies to a certain extent on local medicinal plants to treat several kinds of illness. The inhabitants of the Green Mountain (Al-Jabal Al-Akhdar) of Cyrenaica, Libya have used the medicinal plants grown in the region for thousands of years and have, therefore, provided a rich heritage of folk herbal medicine which have survived through generations (EL-GADI \& El-Mughrabi, 1999). The vegetation of Cyrenaica has long been investigated and a detailed description of the flora of Al-Jabal Al-Akhdar has been produced and published over the last three centuries (Lemaire, 1703; Della-Cella, 1819; Pacho, 1827; Beechey \& Beechey, 1828; Pampanini, 1931; Simpson \& Sandwith, 1941; Boulos, 1972; Brullo \& Furnari, 1979; El-Sherif \& Singh, 1996; El-Mokasabi, 2014; Alaib et al., 2016; SAAED et al,. 2019). Due to their rich vegetation, the floristic composition of the valleys (Wadis) of Al-Jabal Al-Akhdar have lately been devoted special attention; for instance; Wadi Murqus (El-Sherif et al., 1991); Wadi Al-Ashrha (Asker, 1998); Wadi Zaza (El-Barasi et al., 2003); Wadi Al-Kouf (El-MoKasabi, 2014); Wadi Belkaf (Alaib et al., 2016); Wadi Al-Agar (Alaib et al., 2017). However, little is known about the medicinal plants grown in the largest valley of Al-Jabal Al-Akhdar; Wadi Al-Kouf, as no thorough investigation has been carried out. The aim of this study, then, was to record and list the medicinal plant species growing in Wadi Al-Kouf and detect the endemic medicinal species characterizing the valley.

\section{MATERIALS AND METHODS}

\section{The study area}

Wadi Al-Kouf is a valley located in the Cyrenaica district, Eastern Libya, approximately $180 \mathrm{~km}$ north-east of Benghazi, Libya (Fig. 1). It covers the northern slopes and plateau of Al-Jabal Al-Akhdar. The valley extends from the north (the Mediterranean coastline where sand dunes and seasonal wetlands can be found) and gradually ascends to the south to reach an elevation of more than $800 \mathrm{~m}$ asl, with rocky low cliff formation characterizing the sides of the valley (Ben Amer \& Shakman, 2013). The study area lies between latitudes $32.5^{\circ} \mathrm{N}$ and $32.8^{\circ} \mathrm{N}$ and longitudes $21.4^{\circ} \mathrm{E}$ and $22.00^{\circ} \mathrm{E}$, covering an area of $938 \mathrm{~km}^{2}$.

\section{Climate}

A typical Mediterranean climate is predominant in the study area with an average annual rainfall exceeding $630 \mathrm{~mm} / \mathrm{yr}$. Al-Jabal Al-Akhdar is considered the wettest region of Libya receiving an average of $(375-600 \mathrm{~mm})$ of annual precipitation, which increasing with elevation and reaching an average of $1200 \mathrm{~mm} / \mathrm{yr}$ (KAWASMA, 1979). 


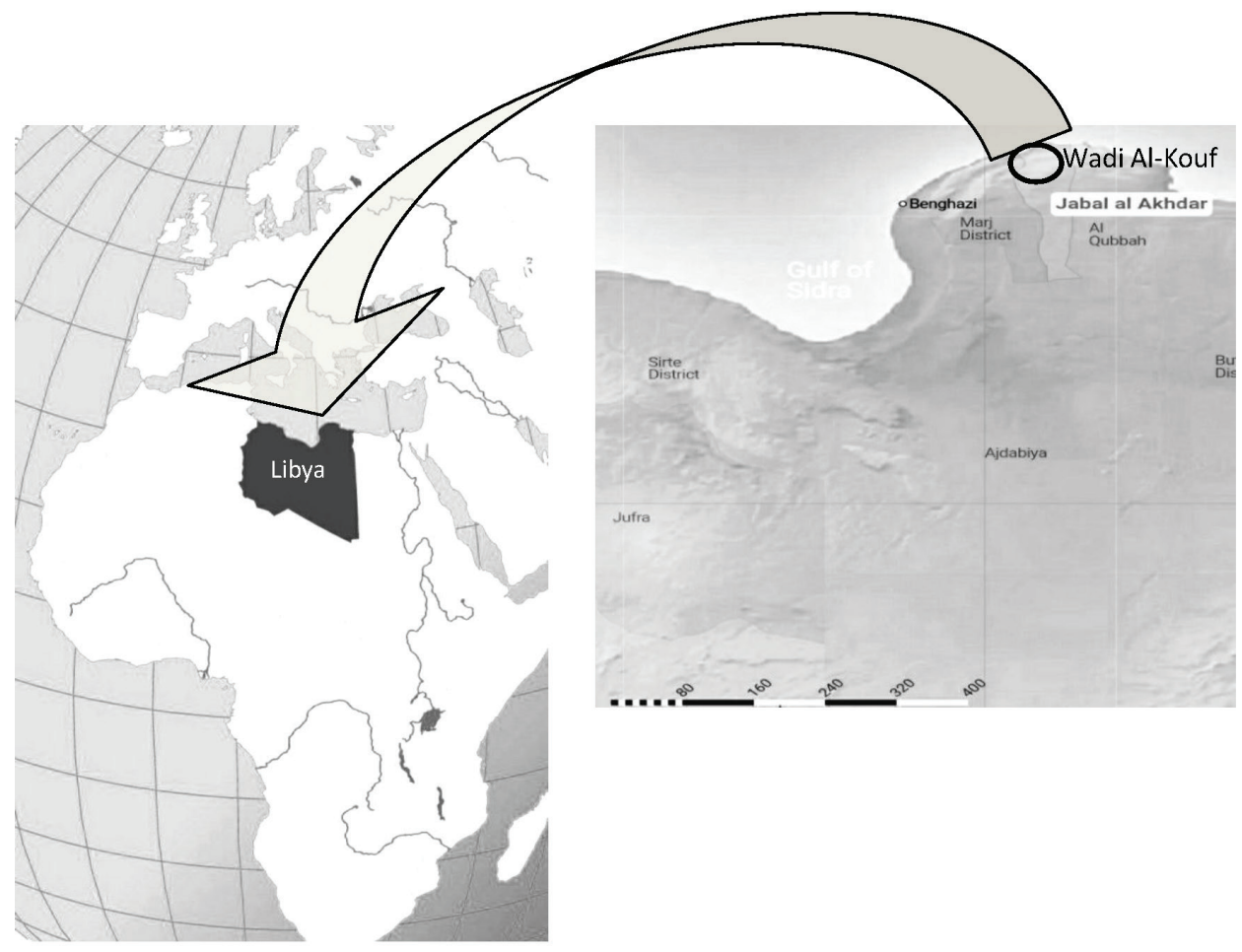

Fig. 1. Geographic position of Wadi Al-Kouf (Map adapted from Google Maps, http://maps.google.com).

Most of the rainfall occurs during the period from October to April, reaching the maximum in December and January (Fig. 2). The lowest average monthly temperature ranges between $4 \mathrm{C}^{\circ}$ in January and $16 \mathrm{C}^{\circ}$ in August, while the highest average monthly temperature ranges between $11.8 \mathrm{C}^{\circ}$ in January and $26.7 \mathrm{C}^{\circ}$ in August (Fig. $3)$. The relative humidity in the valley ranges between $49 \%-55 \%$ in the summer and $65 \%-75 \%$ in the winter. Soil varies at different locations and altitudes; red ferrisialitic carbonate saline clay and red ferrisialitic clay dominate the coastal plains. Rendzinas are found in the lower and middle terraces, with clay-clay loamy-loamy texture, rich in calcium carbonate. Lithic haploxeralfs were the most dominant, followed by Calcic haploxeralfs, Lithic calcixerolls and Typic calcixerolls (SELkhozprom Export, 1980).

\section{Specimen Collection and Identification}

Medicinal plant samples were listed and collected during several field trips during the flowering season (March-April) in 2019. Specimens of the collected plant species were stored at the herbarium of the botany department, Faculty of Sciences, University of Benghazi, Libya. They were identified with the aid of the Libyan Flora Encyclopedia (Flora of Libya) (Ali \& JAFri, 1977; JAFri \& El-GADI, 1986). Plant life forms were also classified into five life forms according to Raunkiaer's system (RAUNKIAER, 1934); (Phanerophytes (Ph), Chamaephytes (Ch), Cryptophytes (C), Hemicryptophytes $(\mathrm{H})$ and Therophytes (Th). Endemic plant species were also detected, recorded and identified. Photographs of all the recorded plant species were taken for documentation. 


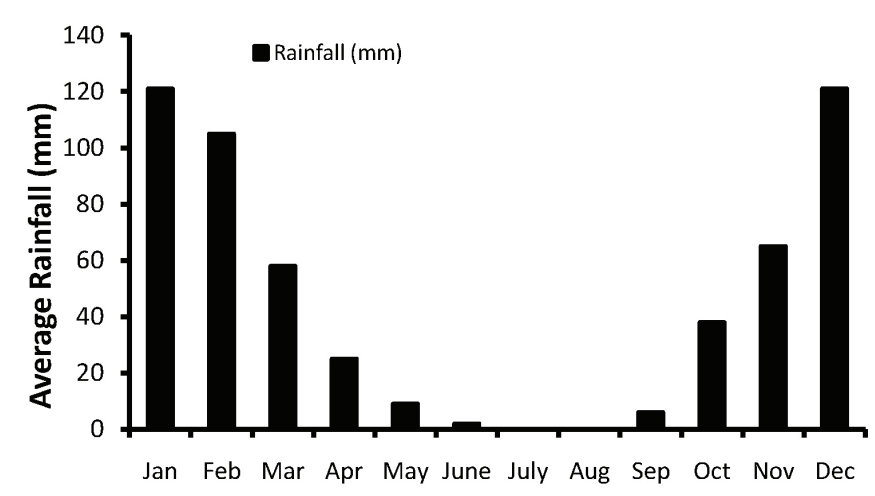

Month

Fig. 2. Average monthly rainfall $(\mathrm{mm})$ in Al-Jabal Al-Akhdar, Libya (Data obtained from www.en.climate-data.org).

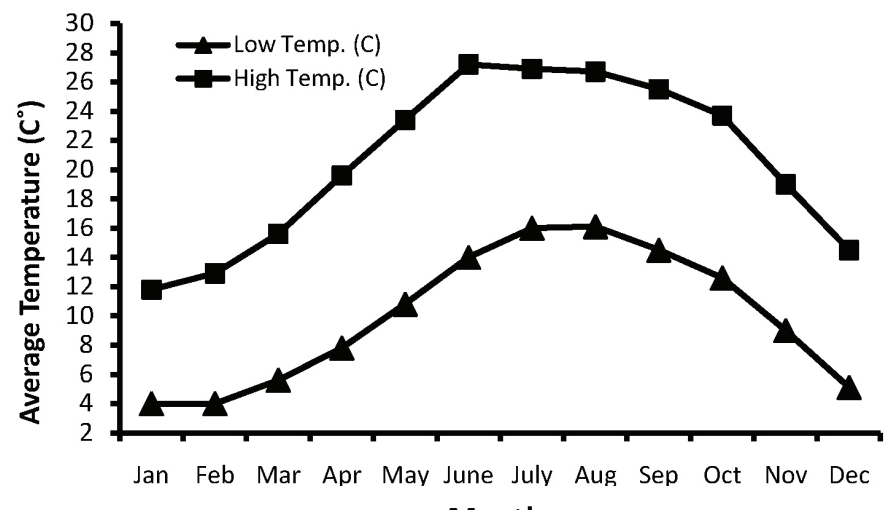

Month

Fig. 3. Average minimum and maximum monthly temperature $\left(\mathrm{C}^{\circ}\right)$ in Al-Jabal Al-Akhdar, Libya (Data obtained from, www.en.climate-data.org).

\section{RESULTS AND DISCUSSION}

Findings of the present study showed the predominance of the Mediterranean flora as the valley was mainly composed of dense scrubland vegetation comprising shrubs and small trees that characterize the Mediterranean biome. Tab. 1 lists the medicinal plant species found in the valley, according to their families, their lifeform, their active ingredients and medicinal uses. The results indicated the presence of a total of 107 medicinal plant species belonging to 49 families and 93 genera. Angiosperms composed the most dominant botanical group with 46 families, 89 genera and 102 species, whereas Gymnosperms were represented by only 3 families, 4 genera, and 5 species (Tab. 2). Dicotyledons were the most presented group of the angiosperms with 38 families, 79 genera and 89 species, whereas Monocotyledons comprised a far less diverse group of medicinal plants in the valley with only 8 families, 10 genera and 13 species.

Asteraceae attained the highest number of genera recorded in the study area with 10 genera and 11 species, followed by Lamiaceae with 9 genera and 9 species, 
Fabaceae was represented by 5 genera and 6 species, whereas Solanaceae had the lowest number of genera, 5, and only 6 species (Tab. 1). Similar results have been reported in previous studies investigating the vegetation of Al-Jabal Al-Akhdar's valleys; Asteraceae was the most dominant family in Wadi Al-Ager, with 46 species (Alaib et al., 2017). It was also the largest family in the Sedy Boras region, represented by 130 species (Alzerbi \& Alaib, 2016) as well as in the Al-mansora region of Al-Jabal Al-Akhdar (Abusaief \& Dakhil, 2013). Along with Poaceae, Asteraceae was found to dominate the valley of Wadi Belkaf with 18 species (AlAiB et al., 2016). Generally, Asteraceae was recorded as the family with the highest number of endemic genera in Al-Jabal Al-Akhdar (El-Darier \& MogasPI, 2009). Overall, Asteraceae has been documented as the most dominant family in Libya representing 237 species (KEITH, 1965; FENG et al., 2013).

Our results revealed that the most represented life-forms of the medicinal plants in the valley were Therophytes (Th) (25.2\%), Phanerophytes (Ph) (25.2\%), Chamaephytes (Ch) (24.3\%), followed by Hemicryptophytes (H) $(15.9 \%)$ and Geophytes $(\mathrm{G})$ (9.3) (Tab. 1; Fig. 4). This biological spectrum is very similar to that of many areas located on the Mediterranean basin. As annuals complete their life-cycle in one single season, Therophytes, the most dominant life-form, are well adapted to the summer droughts and high temperatures that characterize the Mediterranean climate and account for approximately $50 \%$ of the floristic composition present in the biome (Archibold, 1995). Similarly, El-Mokasabi (2014) indicated that Therophytes were dominant (49\%) in Wadi Al-Kouf. The same has been reported for the majority of the valleys investigated in Al-Jabal Al-Akhdar; Therophytes accounted for the highest percentage of contribution to the life form spectrum of medicinal plants across four valleys examined in Al-Jabal Al-Akhdar; Wadi Zaza, Wadi Al Ager, Wadi Jar jar Uma and Wadi Ras Al-Hilal, followed by Phanerophytes and Chamaephytes (MukASSABI et al., 2017). In the present study, like Therophytes, Phanerophytes were represented by an equivalent number of species with a percentage of $25.2 \%$; they were mostly dominated by ever-green sclerophyllous shrubs exhibiting the characteristic morphological features to withstand the dry summer conditions and prevent radiation damage. In similar findings, Phanerophytes were the most dominant representing $50 \%$ of the species found in Wadi Belkaf (Alaib et al., 2016). As they were represented by a percentage of 24.3, Chamaephytes were also considered dominant in this study. Most Chamaephytes of the Wadi Al-Kouf were low growing shrubs having buds and short
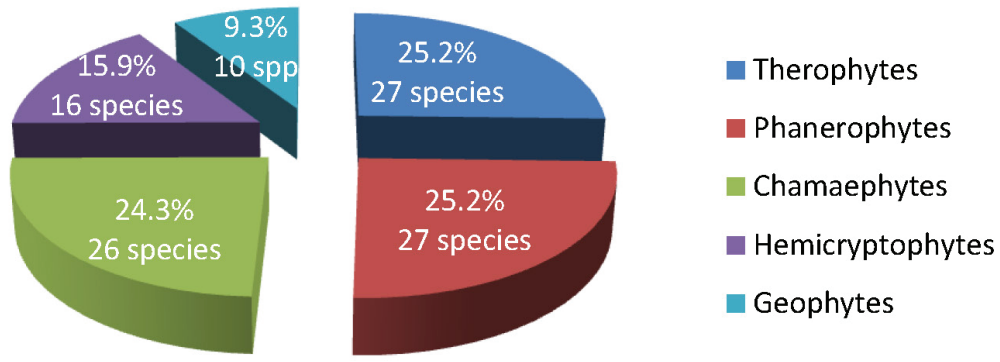

Fig. 4. Life-form spectrum of medicinal plants in Wadi Al-Kouf, Al-Jabal Al-Akhdar (categorized according to Raunkiaer's system; RAUNKIAER, 1934). 


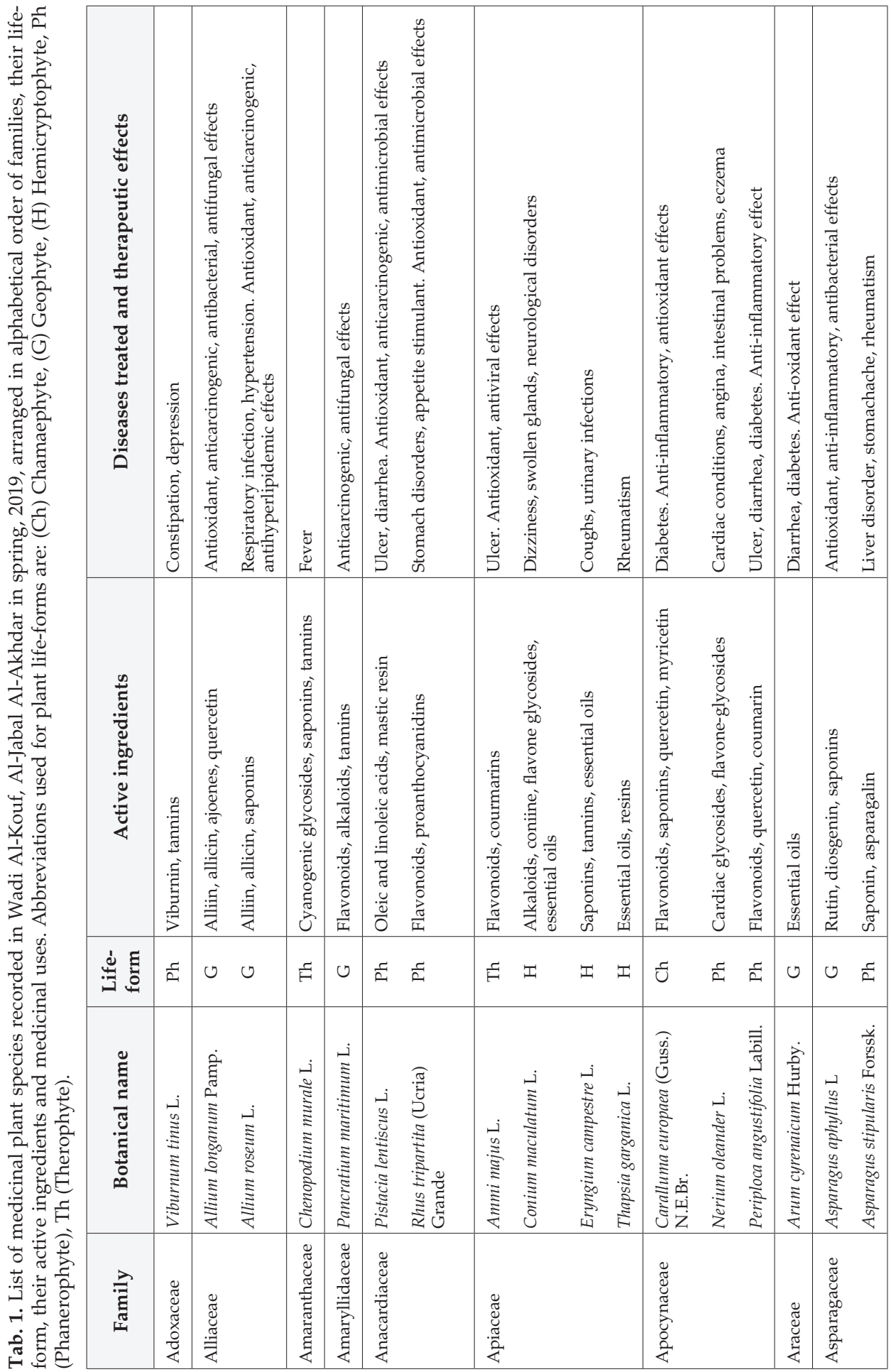




\begin{tabular}{|c|c|c|c|c|c|c|c|c|c|c|c|c|c|c|c|c|}
\hline 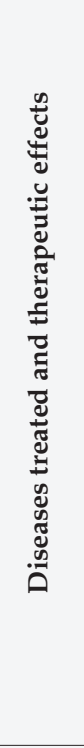 & 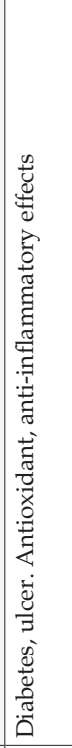 & 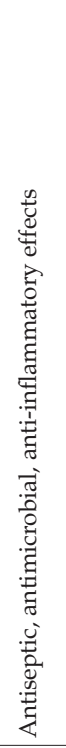 & 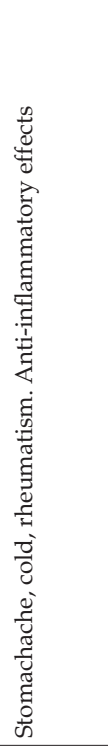 & 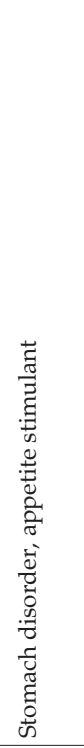 & 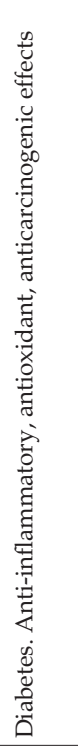 & 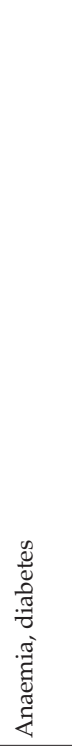 & 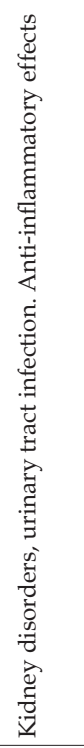 & 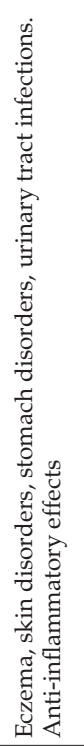 & 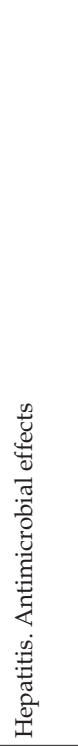 & 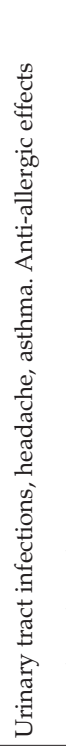 & 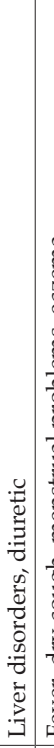 & 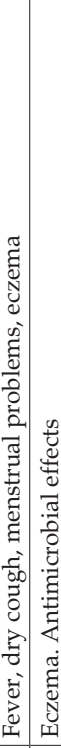 & 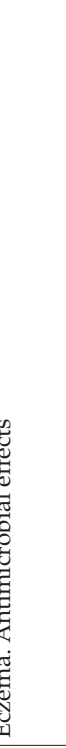 & 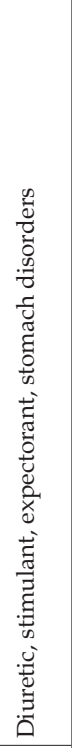 & 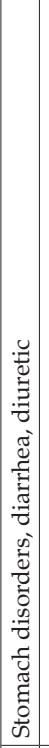 & 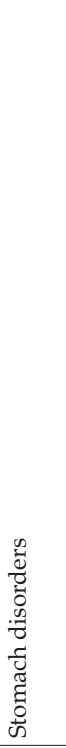 \\
\hline 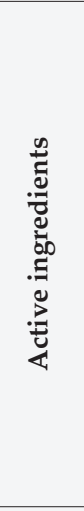 & 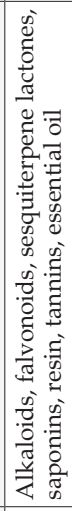 & 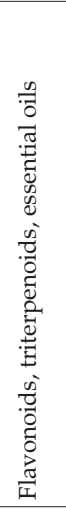 & 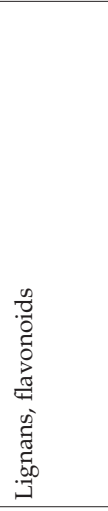 & 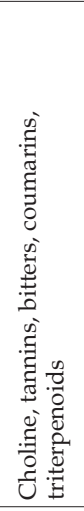 & 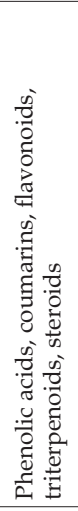 & 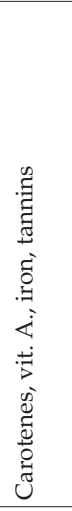 & 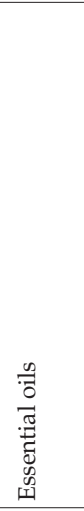 & 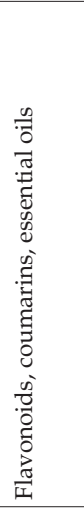 & $\begin{array}{l}\text { F } \\
0 \\
\text { है } \\
\text { है }\end{array}$ & 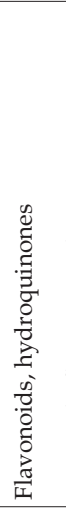 & 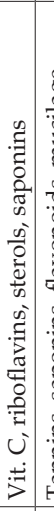 & 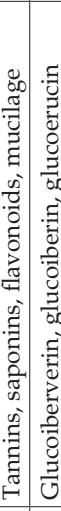 & 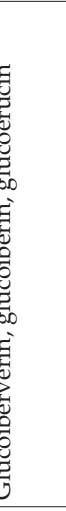 & $\begin{array}{l}\tilde{\xi} \\
\text { है } \\
\text { है } \\
5\end{array}$ & 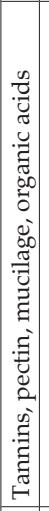 & 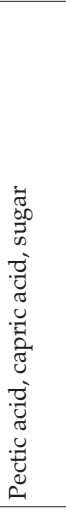 \\
\hline 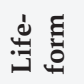 & I & ச & $\mathscr{F}$ & $I$ & $\Xi$ & $\leftleftarrows$ & $\tilde{U}$ & 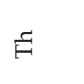 & $\tilde{F}$ & $\tilde{U}$ & $\hat{F} \hat{E}$ & $\Xi F$ & $E$ & $\tilde{U}$ & $\vec{a}$ & שี \\
\hline 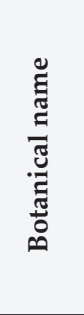 & 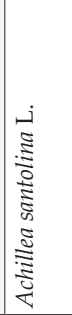 & 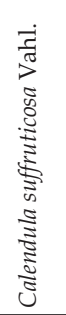 & 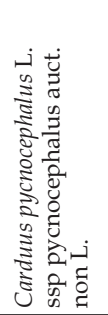 & 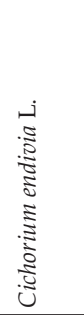 & 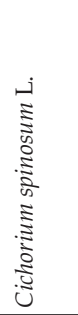 & 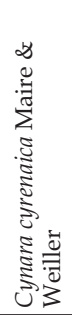 & 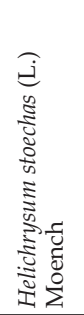 & 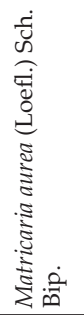 & 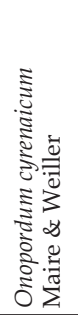 & 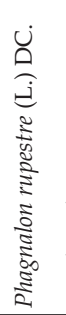 & 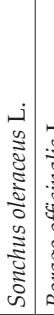 & 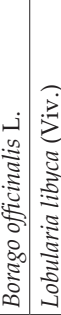 & 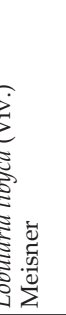 & 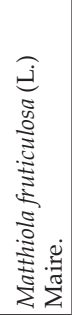 & 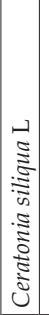 & 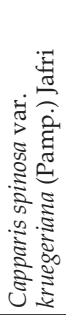 \\
\hline 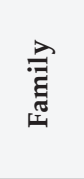 & 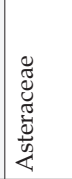 & & & & & & & & & & & 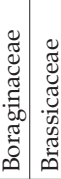 & 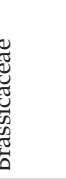 & & 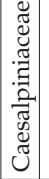 & $\begin{array}{l}\tilde{J} \\
\tilde{\Xi} \\
\tilde{\pi} \\
\tilde{0} \\
\tilde{\tilde{u}} \\
\tilde{u}\end{array}$ \\
\hline
\end{tabular}




\begin{tabular}{|c|c|c|c|c|c|c|c|c|c|c|c|c|c|c|c|}
\hline 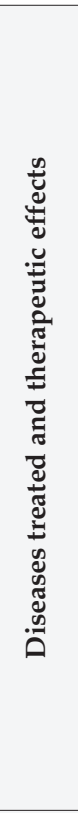 & 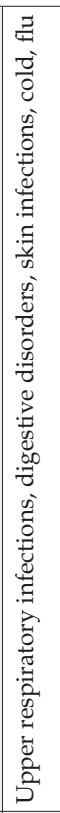 & 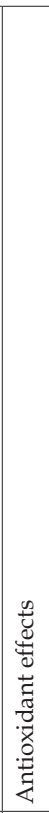 & 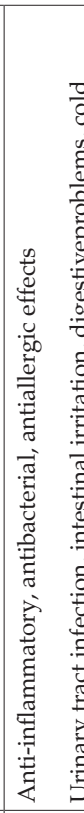 & 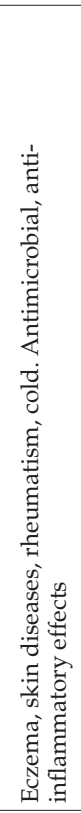 & 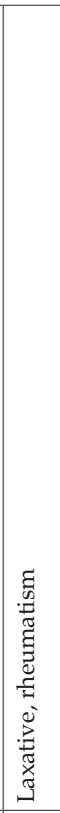 & 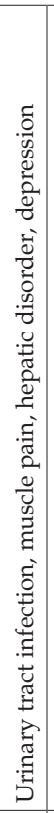 & 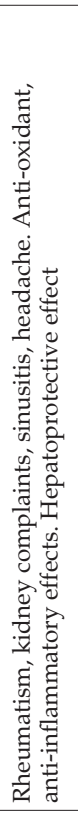 & 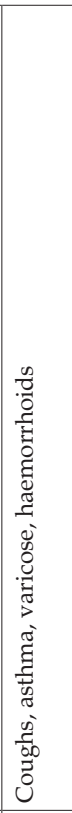 & 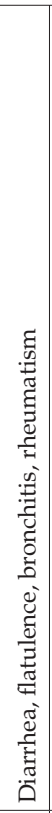 & 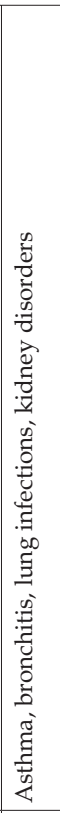 & 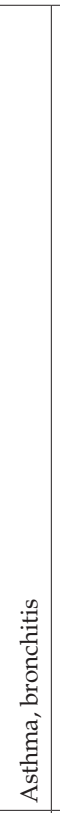 & 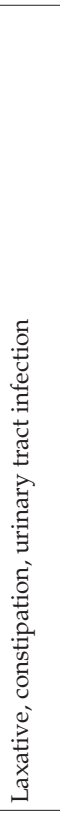 & 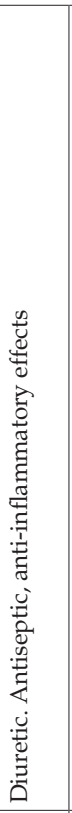 & 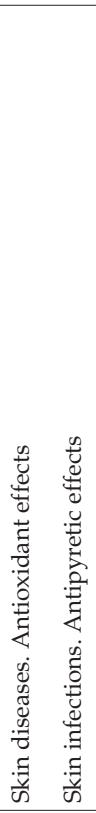 & 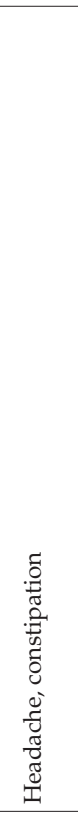 \\
\hline 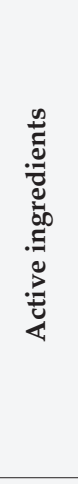 & 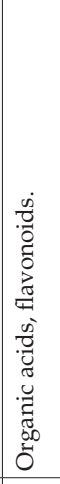 & 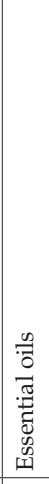 & 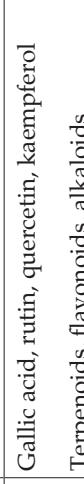 & 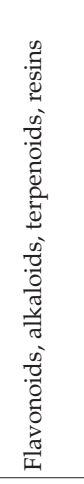 & 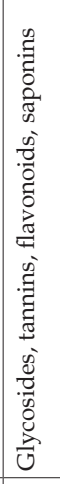 & 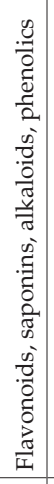 & 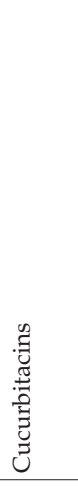 & 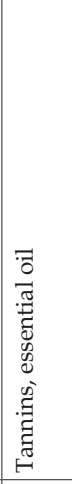 & 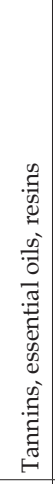 & 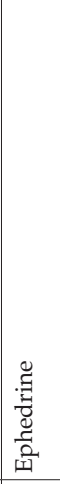 & 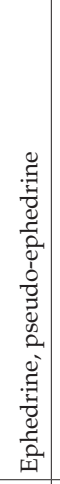 & 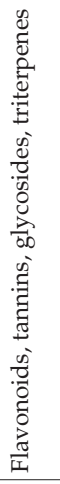 & 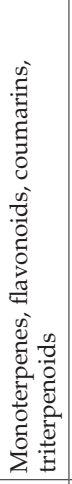 & 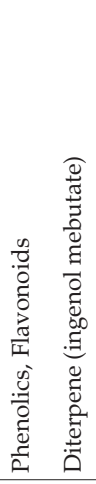 & 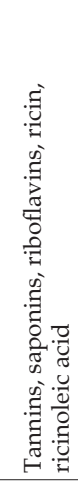 \\
\hline 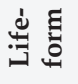 & $\frac{1}{a}$ & 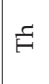 & ह & ฮี & $I$ & $\Xi$ & $\tilde{U}$ & $\frac{E}{\pi}$ & $\underline{a}$ & ச & $\underline{a}$ & $\tilde{E}$ & $\frac{\varepsilon}{2}$ & $\Xi \tilde{E}$ & $\tilde{E}$ \\
\hline 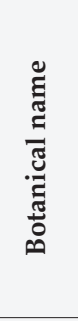 & 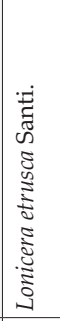 & 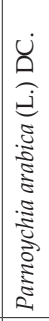 & 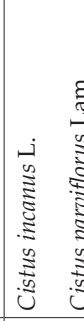 & 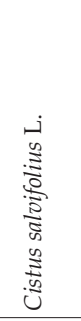 & 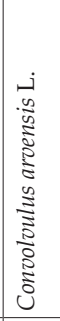 & 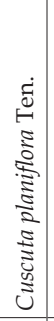 & 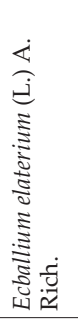 & 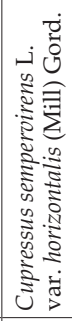 & 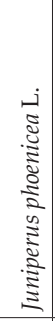 & 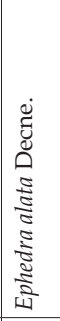 & 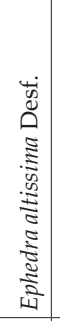 & 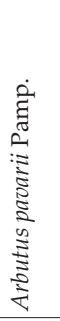 & 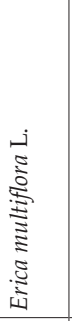 & 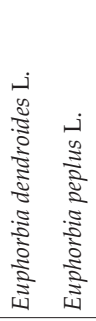 & 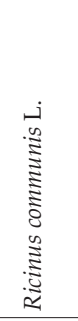 \\
\hline 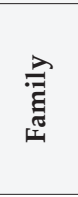 & 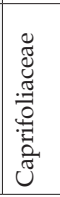 & 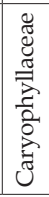 & 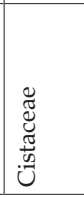 & & $\begin{array}{l}\tilde{J} \\
\tilde{J} \\
\tilde{\sigma} \\
\tilde{J} \\
3 \\
0 \\
0 \\
\tilde{z} \\
0 \\
0\end{array}$ & & 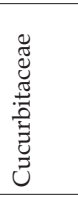 & 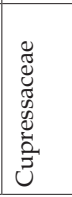 & & 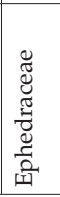 & & 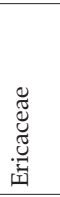 & & 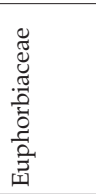 & \\
\hline
\end{tabular}




\begin{tabular}{|c|c|c|c|c|c|c|c|c|c|c|c|c|c|c|c|c|c|c|}
\hline 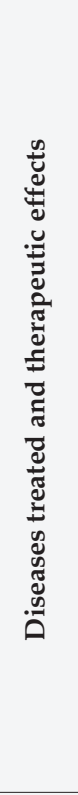 & 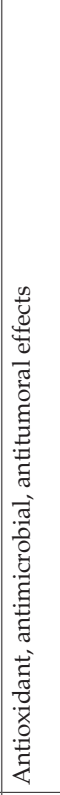 & 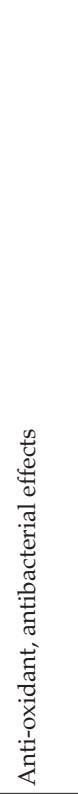 & 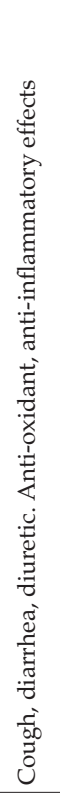 & 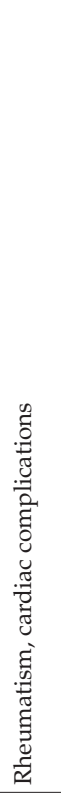 & 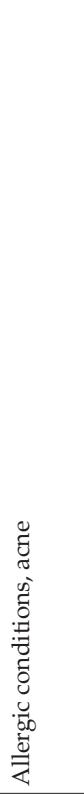 & 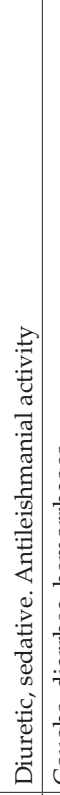 & 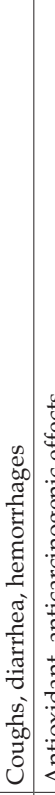 & 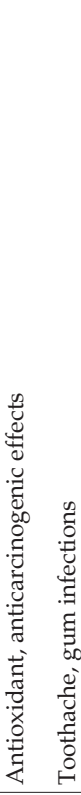 & 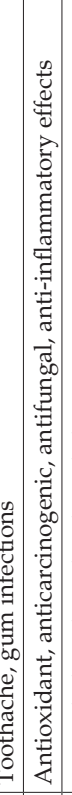 & 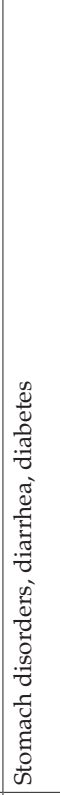 & 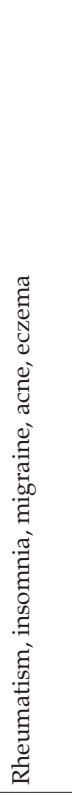 & 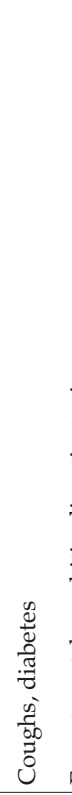 & 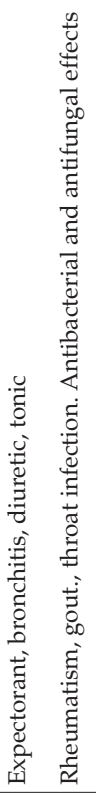 & 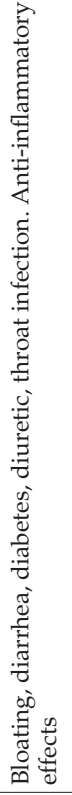 & 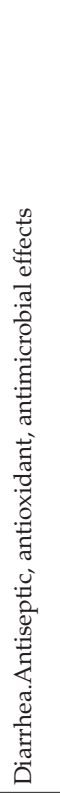 & 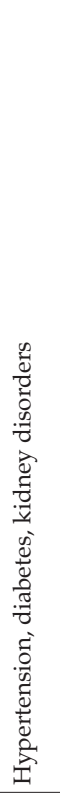 & 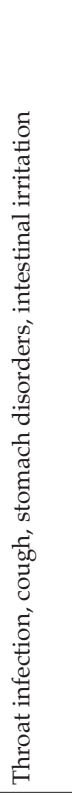 & 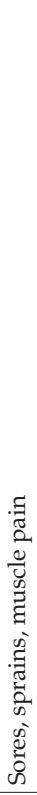 \\
\hline 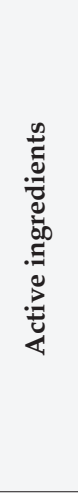 & 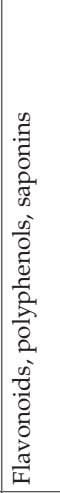 & 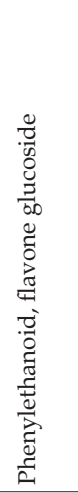 & $\underset{\dot{*}}{u}$ & 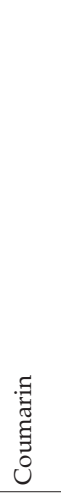 & 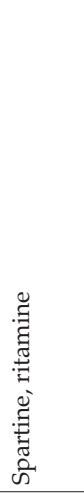 & 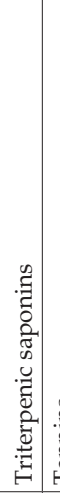 & 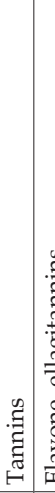 & 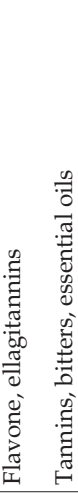 & 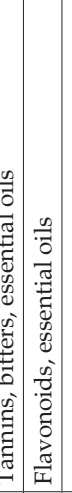 & 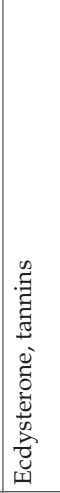 & 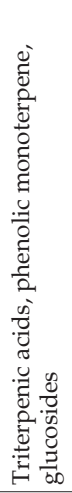 & 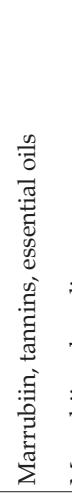 & 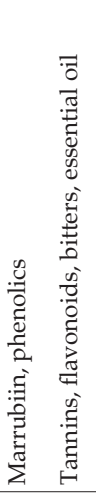 & 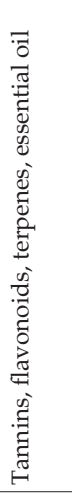 & 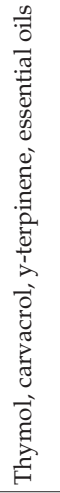 & 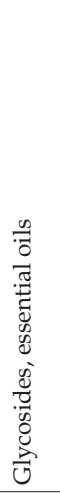 & 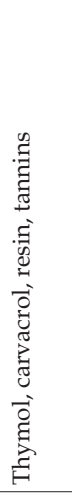 & 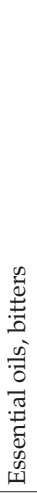 \\
\hline 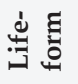 & $\tilde{E}$ & $\tilde{E}$ & 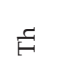 & $\Xi$ & $\underline{\mathscr{L}}$ & $\overrightarrow{C A}$ & $\vec{a} \mid \hat{F}$ & $\leftleftarrows$ & 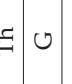 & $I$ & $\tilde{U}$ & I & ச ש & $\tilde{U}$ & $I$ & $\tilde{U}$ & $\tilde{U}$ & $\vec{E}$ \\
\hline 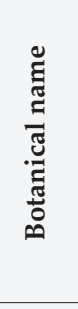 & 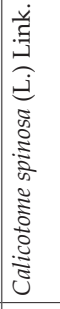 & 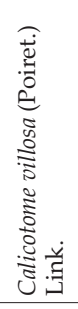 & 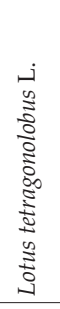 & 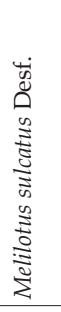 & 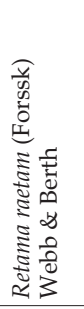 & 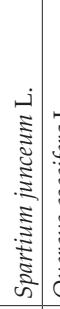 & 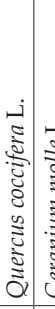 & 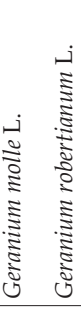 & 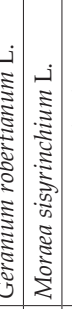 & 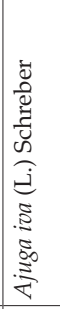 & 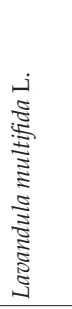 & 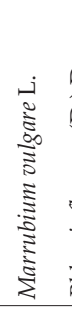 & 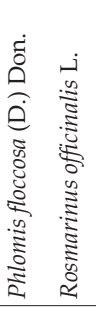 & 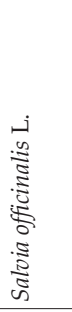 & 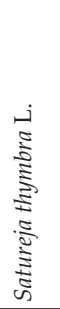 & 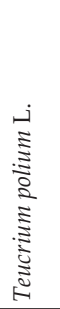 & 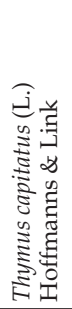 & 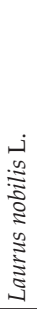 \\
\hline 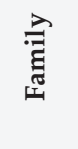 & 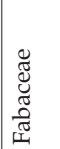 & & & & & & 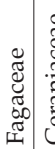 & 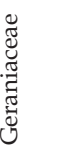 & 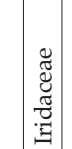 & 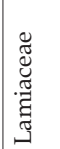 & & & & & & & & \\
\hline
\end{tabular}




\begin{tabular}{|c|c|c|c|c|c|c|c|c|c|c|c|c|c|c|c|c|c|c|}
\hline 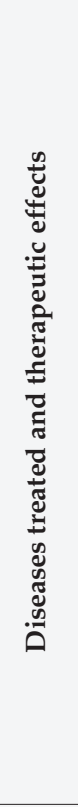 & 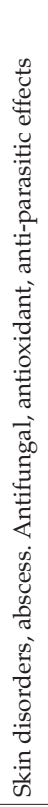 & 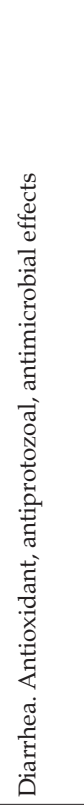 & 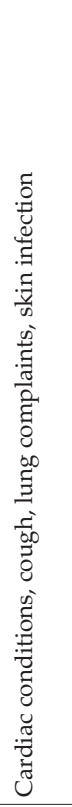 & 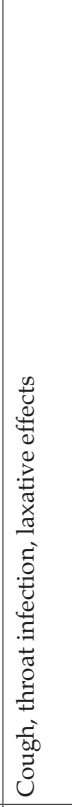 & 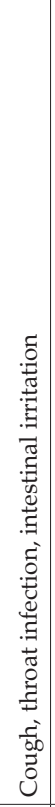 & 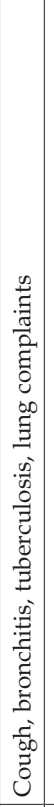 & 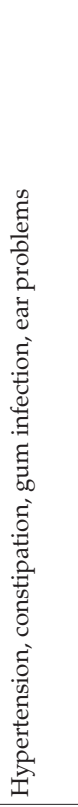 & 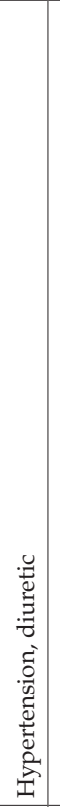 & 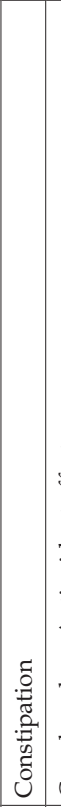 & 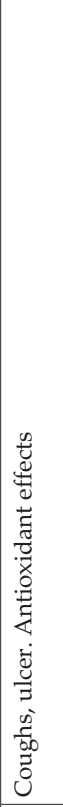 & 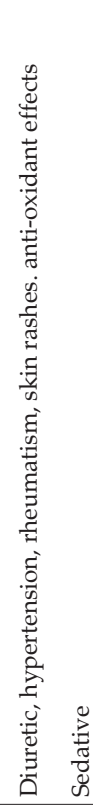 & 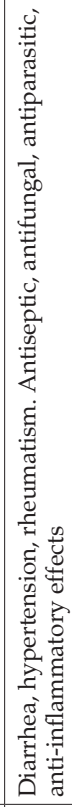 & 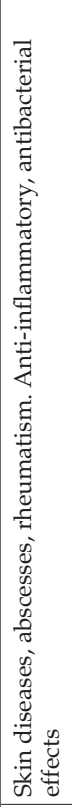 & 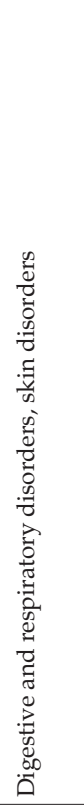 & 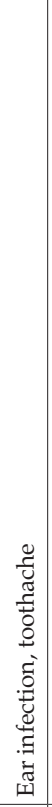 & 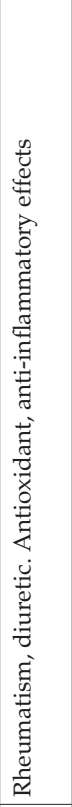 & 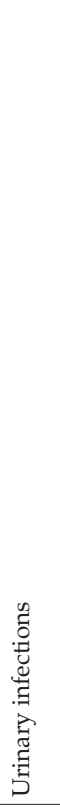 & 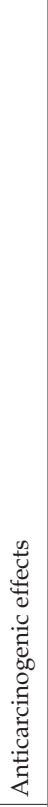 \\
\hline 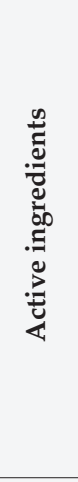 & 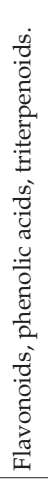 & 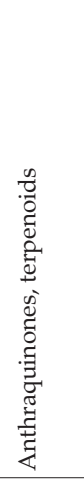 & 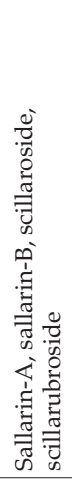 & 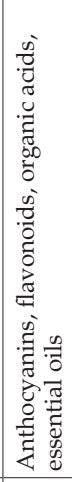 & 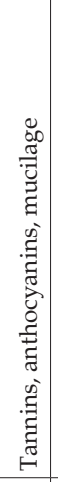 & 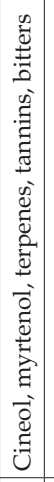 & 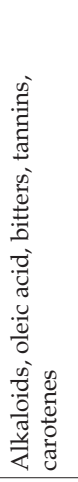 & $\begin{array}{l}\tilde{z} \\
\mathbf{3} \\
\vec{\Xi} \\
\overrightarrow{5} \\
5\end{array}$ & 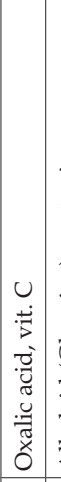 & 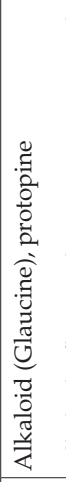 & 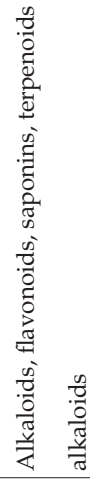 & 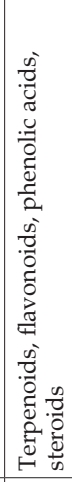 & 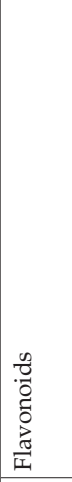 & 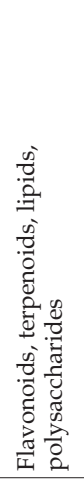 & 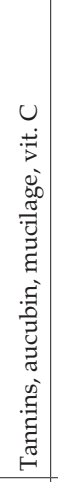 & 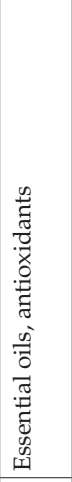 & 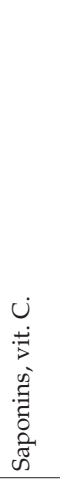 & \\
\hline 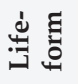 & $\Psi$ & 0 & 0 & $E$ & $E$ & $\frac{\pi}{n}$ & 起 & $\tilde{U}$ & 0 & I & $\Xi \leftleftarrows$ & $\underline{a}$ & $\tilde{U}$ & $I$ & $I$ & $\Xi$ & 工 & $\Psi$ \\
\hline 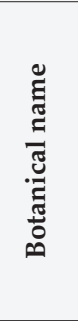 & 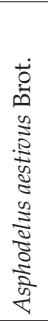 & 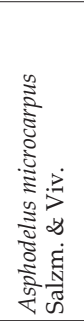 & 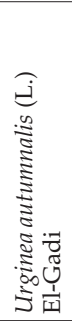 & 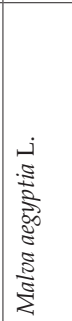 & 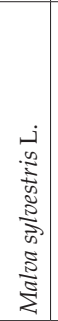 & 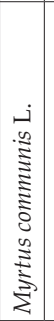 & 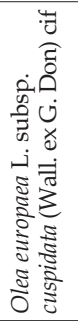 & 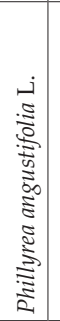 & 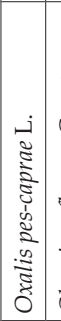 & 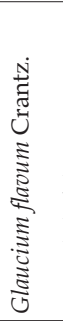 & 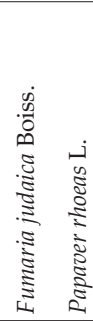 & 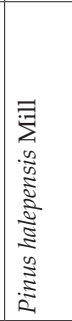 & 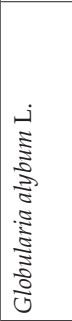 & 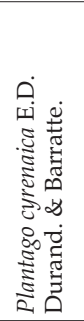 & 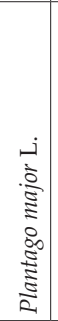 & 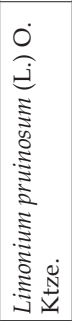 & 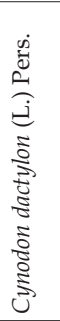 & 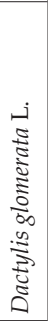 \\
\hline 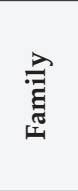 & 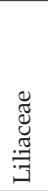 & & & 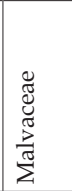 & & 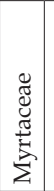 & $\underset{\mathscr{\varpi}}{\mathscr{\Xi}}$ & & 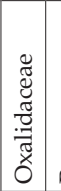 & 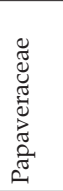 & & 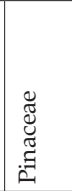 & 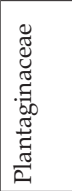 & & & 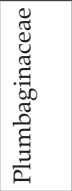 & 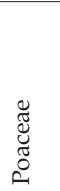 & \\
\hline
\end{tabular}




\begin{tabular}{|c|c|c|c|c|c|c|c|c|c|c|c|c|c|c|c|c|c|c|}
\hline 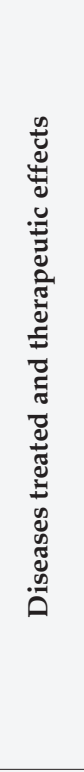 & 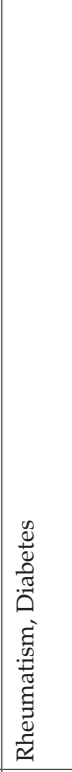 & 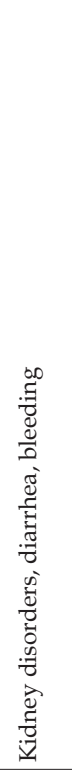 & 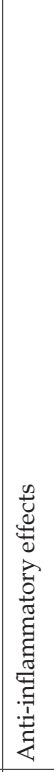 & 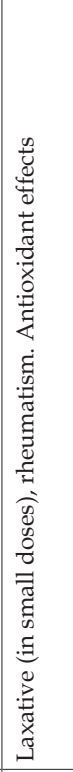 & 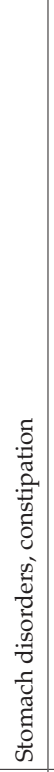 & 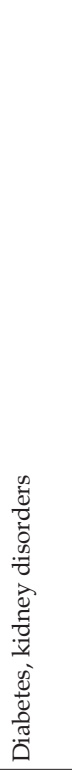 & 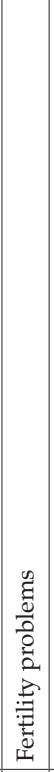 & 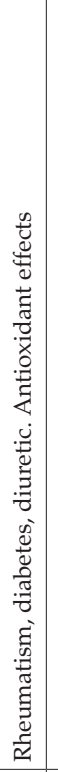 & 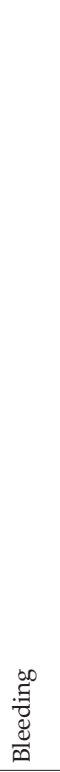 & 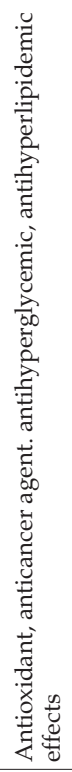 & 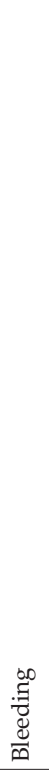 & 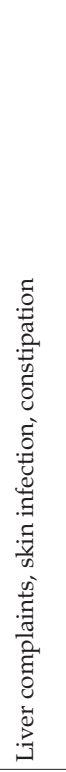 & 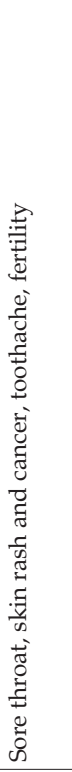 & 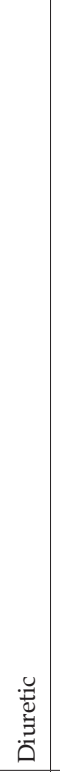 & 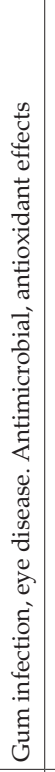 & 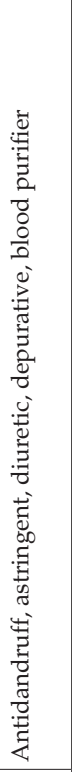 & 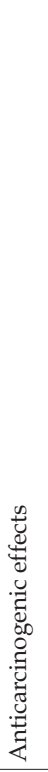 & 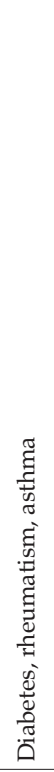 \\
\hline 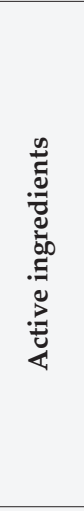 & 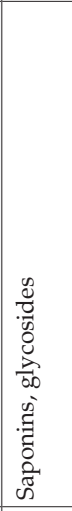 & 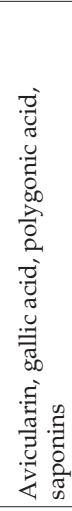 & 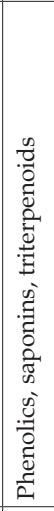 & 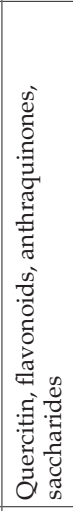 & 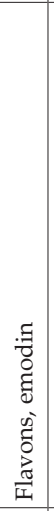 & 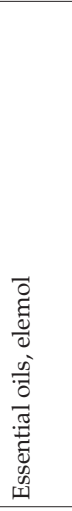 & 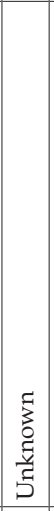 & 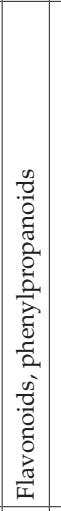 & 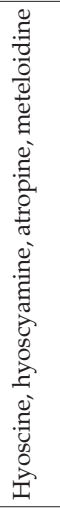 & 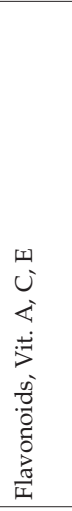 & 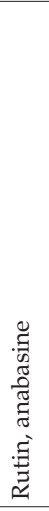 & 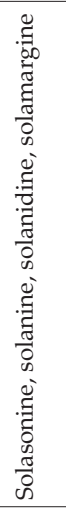 & 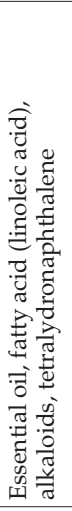 & $\begin{array}{l}\tilde{5} \\
0 \\
0 \\
\tilde{5} \\
5\end{array}$ & 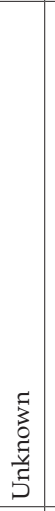 & 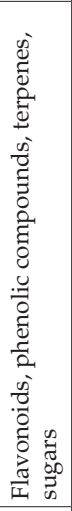 & $\begin{array}{l}0 \\
0 \\
0 \\
0 \\
0 \\
0 \\
0 \\
00 \\
0 \\
0 \\
0 \\
0 \\
0 \\
0 \\
0 \\
0 \\
0 \\
0 \\
0 \\
0 \\
0 \\
0 \\
0 \\
0 \\
0 \\
0 \\
0 \\
\frac{1}{I} \\
\end{array}$ & 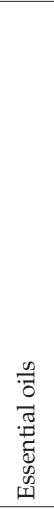 \\
\hline 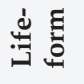 & I & I & 0 & $\frac{E}{a}$ & $\tilde{\Xi}$ & $\tilde{U}$ & ש & $\cup$ & 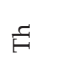 & $\tilde{U}$ & $\underline{\mathscr{A}}$ & $\leftleftarrows$ & 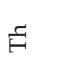 & $\tilde{U}$ & $\tilde{C}$ & F & తే & $\tilde{U}$ \\
\hline 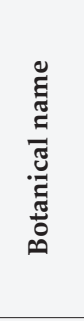 & 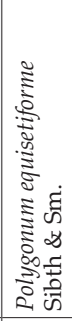 & 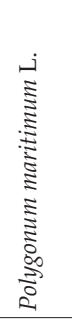 & 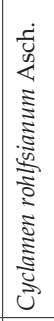 & 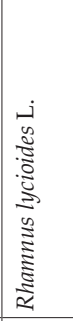 & 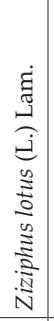 & 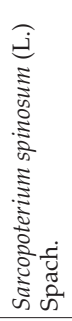 & 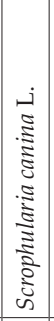 & 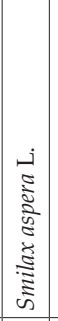 & 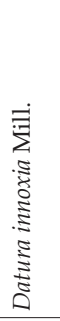 & 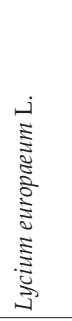 & 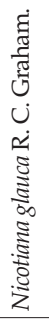 & 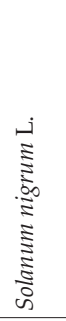 & 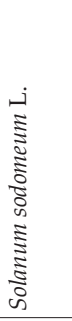 & 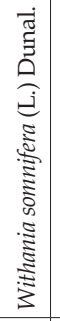 & 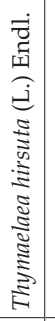 & 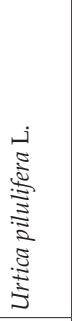 & 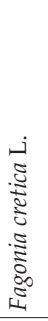 & 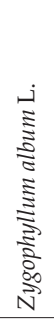 \\
\hline 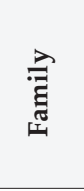 & 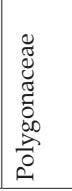 & & 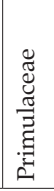 & 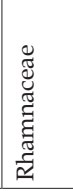 & & $\underset{\widetilde{J}}{\widetilde{J}}$ & 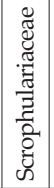 & 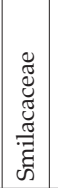 & 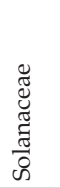 & & & & & & 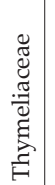 & 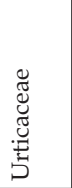 & 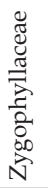 & \\
\hline
\end{tabular}


Tab. 2. Number of families, genera and species for each botanical group, Angiospermae and Gymnospermae, recorded in Wadi Al-Kouf, Al-Jabal Al-Akhdar in spring, 2019.

\begin{tabular}{|c|c|c|c|}
\hline Botanical group & Family & Genus & Species \\
\hline Angiospermae & 46 & 89 & 102 \\
\hline Gymnospermae & 3 & 4 & 5 \\
\hline Total & 49 & 93 & 107 \\
\hline
\end{tabular}

apices close to the ground to be able to survive the unfavorable conditions. Perennials were abundant in the area as their total species number was 74 with a percentage reaching $74.7 \%$ of the total medicinal plants listed. Annuals were represented by 24 species $(25.2 \%)$, whereas only 8 species were classified as biennials, representing $8.1 \%$ of the total plant species found in this study.

The findings of this study clearly indicated that Wadi Al-Kouf had a higher number of medicinal plant species than the adjacent valleys investigated in Al-Al-Jabal Al-Akhdar such as Wadi Zaza, Wadi Al Ager, Wadi Jar jar Uma and Wadi Ras Al-Hilal, in which the total number of medicinal plant species recorded was 49, 36, 41 and 29, respectively (MuKASSABI et al., 2017), whereas fewer medicinal plant species, only 17 of them, were found and listed in Wadi Belkaf (АцаIв et al., 2016), 13 of which were recorded in the present study; Asparagus aphyllus L., Ceratonia siliqua L., Cistus parviflorus Lam., Cistus salvifolius L., Cyclamen rohlfsianum Asch, Helichrysum stoechas (L.) Moench, Juniperus phoenicea L., Marrubium vulgare L., Plantago cyrenaica E.D. Durand. \& Barratte., Quercus coccifera L., Rosmarinus officinalis L., Teucrium polium L., Thymus capitatus (L.) Hoffmanns \& Link. As Wadi Al-Kouf covers an area of approximately $938 \mathrm{~km}^{2}$, it is considered the largest and the widest valley in Al-Jabal Al-Akhdar. It is, therefore, possible that it was the large size that increased the capacity of the valley to host rich and diverse flora. Certain abiotic factors such as temperature, moisture, and soil physical and chemical properties may have positively affected plant growth in the valley and contributed to the observed species richness and the highly diverse flora of medicinal plants characterizing Wadi Al-Kouf. However, such effects are still insufficiently comprehended and need to be thoroughly examined. It should be noted that several human activities pose serious threats to the vegetation of Al-Jabal Al-Akhdar, such as timber exploitation, land reclamation, urbanization, excessive grazing, hunting and touristic activities (EL-BARASI \& SAAED, 2013). However, due to the steep slopes and hills surrounding the valley, many areas of Wadi Al-Kouf are naturally protected from grazing and other human activities as they are out of reach and hard of access. This naturally protective environment may have acted to improve growth conditions for a significant number of plant species, enabling them to survive, reproduce and maintain a complete life cycle in undisturbed environment, consequently, providing a highly diverse cover of flora in the valley. It is worth mentioning that, in the populated area of the valley, numerous medicinal plant species are extensively collected for their medicinal uses and economic values (EL-BARASI \& SAAED, 2013). Information concerning the therapeutic effects and diseases treated by the medicinal plants were obtained from local people and owners of herb shops with the aid of references describing the usage of medicinal plants in Libya (EL-GADI, 1989; El-Gadi \& Bishina, 1992; El-Gadi \& El-Mughrabi, 1999) (Tab. 1). Data revealed that the most frequently used medicinal plants were Ceratonia siliqua L, Cynara cyrenaica Maire \& Weiller, Helichrysum stoechas (L.) Moench , Matricaria aurea (Loefl.) Sch. Bip., 
Tab. 3. List of the endemic medicinal plant species recorded in Wadi Al-Kouf, Al-Jabal Al-Akhdar in spring, 2019, arranged in alphabetical order of families.

\begin{tabular}{|c|l|l|}
\hline & \multicolumn{1}{|c|}{ Species } & \multicolumn{1}{c|}{ Family } \\
\hline 1 & Allium longanum Pamp. & Alliaceae \\
\hline 2 & Arum cyrenaicum Hurby. & Araceae \\
\hline 3 & Cynara cyrenaica Maire. \& Weiller. & Asteraceae \\
\hline 4 & Onopordum cyrenaicum Maire. \& Weiller. & Asteraceae \\
\hline 5 & Capparis spinosa Linn. var. krugeriana (Pamp.) Gafri. & Capparaceae \\
\hline 6 & Cupressus sempervirens L. var. horizontalis (Mill) Gord. & Cupressaceae \\
\hline 7 & Arbutus pavarii Pamp. & Ericaceae \\
\hline 8 & Plantago cyrenaica E.D. Durand. \& Barratte. & Plantaginaceae \\
\hline 9 & Cyclamen rohlfsianum Asch. & Primulaceae \\
\hline
\end{tabular}

Olea europaea L., Rosmarinus officinalis L., Salvia officinalis L., Thymus capitatus (L.) Hoffmanns \& Link., Ziziphus lotus (L.) Lam. Available medicinal plants were commonly used for the treatment of digestive orders $(27.7 \%)$, respiratory system problems $(16 \%)$, rheumatism $(15 \%)$, skin infection $(13.5 \%)$, diabetes $(11.7 \%)$, cancer $(8.5 \%)$, cold and flu $(7.5 \%)$. There is an increasing concern that irresponsible resource exploitation may lead to vegetation deterioration threatening the existence of the medicinal plants in the valley.

In total, there are 134 endemic plant species in Libya (BEentJe et al., 1994). Due to its distinctive physiographic and climatic barriers, Al-Jabal Al-Akhdar represents an exceptional refuge for numerous endemic plant species (AL-SODANY et al., 2003), as it is known to host most of the endemic species of the country (Boulos, 1997), approximately $60 \%$ of Libya's endemic species (RADFORD et al., 2011). In all, in Al-Jabal Al-Akhdar 59 endemic plant species have been recorded (QAISER \& EL-GADI, 1984), 29 of them in Wadi Al-Kouf (El-MokasabI, 2014). Nine of the 107 medicinal plants listed in the present study were classified as endemic species (Tab. 3). Five of the 9 reported endemic medicinal plants; Allium longaunm Pamp., Arbutus pavarii Pamp., Arum cyrenaicum Hurby, Cyclamen rohlfsianum Asch., Onopordum cycrenaicum Marie \& Weiller were also found in the neighboring Wadi Al-Ager and listed as endemic species along with an additional 13 species (Alaib et al. 2017). Similarly, the five endemic species recorded in Wadi Belkaf (Alaib et al., 2016) were also found and listed in this study; Allium longanum Pamp., Arbutus pavarii Pamp., Arum cyrenaicum Hurby., Cyclamen rohlfsianum Asch., Plantago cyrenaica E.D. Durand. \& Barratte.

Two of the endemic medicinal plants listed in the present study, Arbutus pavarii Pamp. and Cupressus sempervirens L. var. horizontalis (Mill) Gord., were classified by the Libyan conservation program as endangered species (EL-BARASI \& SAAED, 2013; Mosallam et al., 2017). Based on its current observation status, Arbutus pavarii (Fig. 5) has also been placed in the Red List by the International Union for Conservation of Nature (IUCN, 2021) as vulnerable and threatened with global extinction (IUCN, 1998), whereas Cupressus sempervirens L. was categorized as (Least Concern) (FARJON, 2013). Several regulations and laws regarding management of natural resource and conservation of natural vegetation have been passed and issued by the Libyan authorities in the past four decades; however, they have not been properly implemented. For instance; Al-Kouf National Park was one of the early conservation projects in Libya (Ben Amer \& Shakman, 2013). This natural reserve was established in 


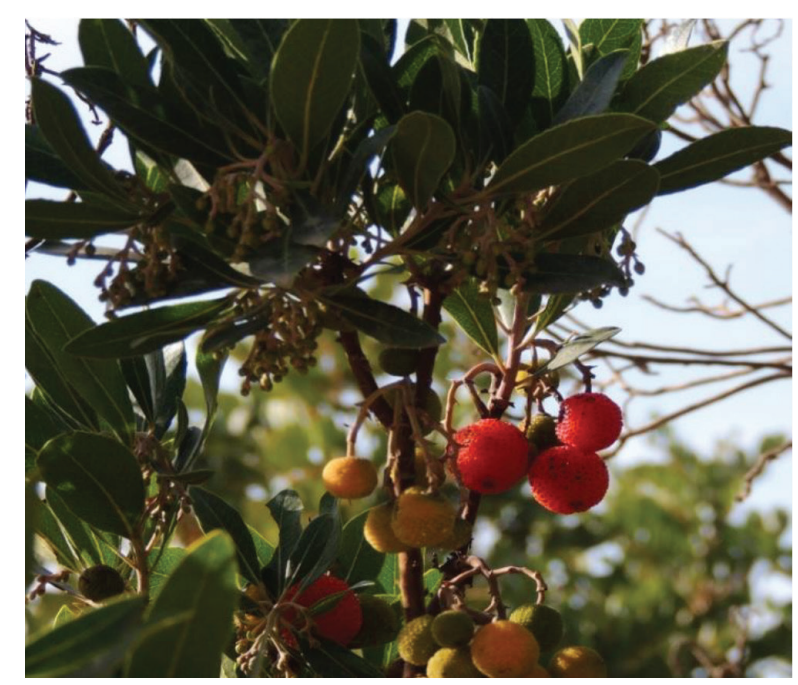

Fig. 5. Arbutus pavarii Pamp. Al-Jabal Al-Akhdar, Libya. Photograph by M. Al-Traboulsi, 2020.

1979 to conserve biodiversity and provide protection for natural flora and fauna living in a protected area of approximately 9000 ha of the valley. Unfortunately, most of the conservation attempts have had a little effect on preserving the site and failed to monitor human activities, the main cause of vegetation disturbance, within the boundaries of the valley.

Based on the findings of the present study, it can be concluded that Wadi Al-Kouf has a higher medicinal plant species richness than neighbouring valleys in Al-Jabal Al-Akhdar. However, given the fact that two of the endemic medicinal plants have been designated endangered species, there is a growing concern that they may become extinct in the near future as they are not yet protected. Therefore, immediate measures should be taken to activate the conservation programs designed to preserve the unique species diversity of Wadi Al-Kouf and provide protection for medicinal, rare and endemic species. The conservation efforts should also involve the local community living in the valley. Human activities should be regulated, not only by legal regulations, but also by increasing the inhabitants' awareness of the value of the endemic medicinal plants and the destructive impact of biodiversity loss on the region. Frequent documentation of the natural vegetation, particularly, medicinal and endemic plants, is strongly recommended.

Received April 25, 2021

\section{REFERENCES}

Abusaief, H. M. \& Dakhil, A. H., 2013: The floristic composition of rocky habitat of Al-Jabal Al-Akhdar, Libya. New York Science Journal 6(5), 34-45.

Alaib, M. A., Elbakkosh, A. M., Abdulati, Y. B., Gadelmola, A. S. \& Elmhafdi, A. M., 2016: Preliminary investigation of the vegetation of Wadi Belkaf-Bata, Al-Jabal Al-Akhdar. Libyan Journal of Science 4(1), 20-27.

Alaib, M., El-Sherif, I. \& Al-Hamedi, R., 2017: Floristic and ecological investigation of Wadi Al-Agar in Al-Jabal Al-Akhdar, Libya. Journal of Science and its applications 5(1), 57-61. 
Ali, S. I. \& JAFri, S. M., 1977: Flora of Libya. Vols. 1-24. Department of Botany, Al-Fateh University, Tripoli, Libya.

Al-Sodany, Y. M., Shehata, M. N. \& Shaltout, K. H., 2003: Vegetation along an elevation gradient in Al-Jabal Al-Akhdar, Libya. Ecologia mediterranea 29, 125-138.

Alzerbi, A. \& Alaib, M., 2017: Study of vegetation in Sedy Boras region in Al-Jabal Al-Akhdar-Libya. Journal of Environmental Science and Engineering 1(1), 67-72.

Archibold, O. W., 1995: Ecology of world vegetation. Champman \& Hall. London.

Asker, A. M., 1998: Vegetation and flora of Wadi Al-Ashrha (Al-Jabal Al-Akhdar). M. Sc. Thesis. University of Garyounis, Benghazi, Libya.

Beechy, F. W. \& Beechy, H. W., 1828: Proceeding of the expedition to explore the northern coast of Africa, from Tripoli eastward, London.

BeentJe, H. J., Adams, B. \& Davis, S. D., 1994: Regional overview: Africa. In: Davis, S. D., Heywood, V. H. \& Hamilton, A. C. (eds). Centers of plant diversity. Oxford.

Ben Amer, I. \& Shakman, E., 2013: Economic and Social survey of El kouf National Park in Libya. MedMPAnet project, Tunis: 29p.

Boulos, L., 1972: Our present knowledge of the flora and vegetation of Libya. Webbia. 26, 366-400

Boulos, L., 1997: Endemic Flora of the Middle East and North Africa, in: BaraKat, H. N. \& Hegazy, A. K (Eds.), Review in Ecology, Desert Conservation and Development. IDRC/CRDI. UNESCO/ South Valley University, Cairo. pp. 229-260.

Brullo, S. \& Furnari, F., 1979: Taxonomic and nomenclatural notes on the flora of Cyrenaica "Libya." Webbia 34(1), 155-174.

Della-Cella, P., 1819: Viggio da Tripoli di Barberia alle frontiere occidentali della Egitto.

El-Barasi, Y., El-Sherif, I. \& Gawhari, A., 2003: Checklist and analysis of the flora and vegetation of Wadi Zaza at Al-Jabal Al-Akhdar (Cyrenaica, Libya). Bocconea 16, 1091-1105.

El-BARASI, Y. \& SAAED, M., 2013: Threats to plant diversity in the North Eastern part of Libya (El-Jabal El-Akhdar and Marmarica plateau). Journal of Environmental sciences and Engineering 2, 41-58.

El-Darier, S. M. \& El-Mogaspi, F. M., 2009: Ethnobotany and relative importance of some endemic plant species at El-Jabal El-Akhdar region (Libya). World Journal of Agricultural Sciences 5(3), 353-360

EL-GADI, A. \& Bishina, S., 1992: Usages of some plants in Libyan folk medicine. I. Dar Al-Kutub. Benghazi.

EL-GADI, A., 1989: Usages of some plants in Libyan folk medicine. II. Dar Al-Kutub. Benghazi.

El-Gadi, A. \& El-Mughrabi, M. 1999: Usages of some plants in Libyan folk medicine. III. Dar Al-Hekma. Tripoli.

El-Mokasabi, F. M., 2014: Floristic composition and traditional uses of plant species at Wadi Alkuf, Al-Jabal Al-Akhdar, Libya. American Eurasian Journal of Agricultural and Environmental Sciences 14(8), 685-697.

El-Sherif, I., El-Barasi, Y., Mugasabi, M., El-Adrawi, M., Shakmahk, Y. \& Gomma, M., 1991: A contribution to the flora of Wadi Murquis (Al-Jabal El-Akhdar, Libya). Acta Botanica India 19, 232-235.

EL-Sherif, I. \& Singh, V., 1996: Vegetation and flora of Benghazi on the Mediterranean cost of Libya. Advances in Plant Science Research 3, 1-68.

FARJon, A., 2013: Cupressus sempervirens. The IUCN Red List of Threatened Species 2013: e. T32518A2821211. Accessed on 10 December 2021.

FEnG, Y., LeI, J., XU, X. \& PAN, B., 2013: Composition and characteristics of Libya flora. Archives of Biological Sciences 65(2), 651-657.

IUCN, 2021: The IUCN Red List of Threatened Species. Version 2021-3. https:/ / www.iucnredlist.org. Accessed on 10 December 2021.

JAFri, S. M. \& El-Gadi, A., 1986: Flora of Libya. 25-144. Department of Botany, Al-Fateh University, Tripoli, Libya.

Kawasma, Y., 1979: Climate of Kouf National Park. Kouf National Park. Report to the Government of Libya.

Kегтн, H., 1965: A preliminary check list of Libyan flora. 2 vols. Ministry of Agriculture and Agrarian Reform. London.

Lemair, F., 1703: Les antiquites de la Cyrenaique ouil est aussi question du "seltion" que M. Bonnet areconnu etra le Phlomis floccosa. France. 
Lewis, W. H. \& Elvin-Lewis, M., 2003: Medical Botany: Plants affecting human health. 2nd ed. Wiley, New Jersey.

Mosallam, H., Hashim, S. \& Sergiwa, S., 2017: A size distribution of some endangered plant species, Al-Jabal Al-Akhdar, Libya. Egyptian Journal of Botany. 57(1), 181-197.

Mukassabi, T., Thomas, P. A. \& Elmogasapi, A., 2017: Medicinal plants in Cyrenaica, Libya: existence and extinction. Biological Diversity and Conservation. 10(2), 183-192.

PAсно, I. R., 1827: Relation d'un voyage dans la Marmarique, la Cyrenaique, et les Oasis d' Audjelah et de Maradeh, Texted $4 \mathrm{me}$ partie, Oasis Meridionale, 4 Planches. Liv. VIII. IX. X. (et derniere) Folio. Each 12s-L'onvrage Cotnplle'te, 2 vols, 4to et 1 vol. folio $81.8 \mathrm{~s}$.

Pampanini, R., 1931: Prodomo della Crenaica Minstero Dello Colonie, Forli.

Podlech, D., 2001: Herbs and healing plants of Britain and Europe. Collins Nature Guide. Harper Collins publishers, UK.

QAISER, M., \& El-GADI, A., 1984: A critical analysis of the flora of Libya. Libyan Journal of Science 13, 31-40.

RadFord, E., Catullo, G. \& Montmollin, B., 2011: Important plant areas in the south and east Mediterranean region. Priority sites for conservation ISBN 978-2-8317-13337-3.

Raunkiaer, C., 1934: The plant life forms and statistical plant geography. Clarendon Press, Oxford. Pp. 632.

SaAed, M., El-Barasi, Y. \& Rahil, R., 2019: Our present knowledge about the history and composition of the vegetation and flora of Libya. Webbia 74(2), 325-338.

SELKHOZPROM EXPORT, 1980: Soil studies in the eastern zone of Libya. Secretariat for Agriculture. Tripoli, Libya. Report to the Government of Libya.

Simpson, D. \& SANDwith, N., 1941: Addition to the flora of Cyrenaica. Journal of Botany 79, 33-44.

White, L. \& Foster, S., 2000: The herbal drugstore. Rodale Inc. London.

WORLD CONSERVATION MONITORING CENTRE, 1998: Arbutus pavarii. The IUCN Red List of Threatened Species 1998: e.T30323A9535904. Accessed on 10 December 2021. 